\title{
PERCEPÇÕES SOBRE CUIDAR DE SI, PROMOÇÃO DA SAÚDE E SOBREPESO ENTRE ACADÊMICOS DE ENFERMAGEM
}

\author{
Perceptions of Self-care, health promotion and overweight among nursing students \\ Percepciones sobre el cuidado de si mismo, promoción de la salud y sobrepeso en \\ estudiantes de enfermería
}

\begin{abstract}
RESUMO
Pesquisa qualitativa, Convergente Assistencial (PCA), desenvolvida com 42 acadêmicos de enfermagem, em uma Universidade Pública da Região Sul do Brasil. Objetivou identificar as percepções dos acadêmicos de enfermagem sobre o cuidar de si, para a promoção da saúde e a evitabilidade do sobrepeso. A coleta dos dados deu-se durante quatro encontros, através de dinâmicas de grupo, confecções de cartazes, atividades lúdicas, utilização de instrumento. A análise dos dados seguiu PCA. Categorias: Atividades físicas; Alimentação saudável; Ambiente familiar; Autoestima e autoimagem. Resultados: Os acadêmicos reconhecem a importância de Cuidar de Si; apresentam conhecimento e relacionam à temática; percebem o Cuidar de Si como forma de manter peso e/ou buscar peso adequado. As contribuições estão no sentido de novas reflexões sobre a realidade dos acadêmicos, e subsídios para inovação do ensino e aprendizagem, sensibilização dos futuros profissionais da área.
\end{abstract}

Palavras-chave: Autocuidado. Hábitos. Peso Corporal. Estudantes de Enfermagem. Enfermagem.

\begin{abstract}
Qualitative research, Convergent Assistance (PCA), developed with 42 nursing students in a public university in southern Brazil. Aimed at identifying students' the perceptions of nursing students about the Care of You, to promote health and prevent overweight. Data collection took place during four meetings, through group dynamics, production of posters, play activities, use of instrument. Data analysis followed by PCA. Categories: Physical activity, healthy food, family environment, self-esteem and selfimage. Results: academics acknowledge the importance of caring for oneself, present knowledge and relate to the theme; realize the Care of Yourself as a way to maintain weight and / or seek appropriate weight. Contributions are to new thinking about the reality of the students, and grants for innovative teaching and learning, awareness of future professionals.
\end{abstract}

Keywords: Self care. Habits. Body weight. Students, Nursing. Nursing.

\section{Resumen}

Investigación cualitativa, Convergente Asistencial (PCA), desarrollada con 42 estudiantes de enfermería en una universidad pública en el sur de Brasil. El objetivo fue identificar las percepciones de los estudiantes de enfermería sobre el cuidado consigo mismo, para promover la salud y prevenir el sobrepeso. La recopilación de datos tuvo lugar durante cuatro sesiones: a través de dinámicas de grupo, de la producción de carteles, de actividades lúdicas, del uso de instrumentos. El análisis de datos siguió PCA. Categorías: actividades físicas, alimentación saludable, ambiente familiar, autoestima y autoimagen. Resultados: los académicos reconocen la importancia de cuidar de si mismos; presentan conocimientos y los relacionan con el tema; perciben el Cuidar de Si mismo como una manera de mantener el peso y / o buscar un peso adecuado. Las contribuciones son a un nuevo pensamiento sobre la realidad de los estudiantes, y subvenciones para la enseñanza y aprendizaje innovadoras, la sensibilización de los futuros profesionales.

Palabras clave: Autocuidado. Hábitos. Peso corporal. Estudiantes de Enfermería. Enfermería.

\footnotetext{
'Doutoranda do Programa de Pós-Graduação em Enfermagem da Universidade Federal de Santa Catarina (UFSC). Mestre em Enfermagem pelo Programa de PósGraduação em Enfermagem (UFSC). Bolsista do CNPQ (Conselho Nacional de Desenvolvimento Científico e Tecnológico). Membro do Grupo de Pesquisa C\&C Cuidando e Confortando, da UFSC. Florianópolis - SC. Brasil. Email: fabisebold@gmail.com;Pós-Doutora pela University of Alberta. Doutora e Mestra pela Universidade Federal de Santa Catarina (UFSC). Professora do Departamento de Enfermagem e do Programa de Pós-Graduação da UFSC. Líder do Grupo de Pesquisa C\&C - Cuidando e Confortando, da UFSC. Florianópolis - SC. Brasil. Email: radunz@nfr.ufsc.br ;3Pós-Doutora pela Universidade de São Paulo (USP Ribeirão Preto). Doutora e Mestra pela Universidade Federal de Santa Catarina. Professora do Departamento de Enfermagem e do Programa de Pós-Graduação da Universidade Federal de Santa Catarina. Vice-Líder do Grupo de Pesquisa C\&C - Cuidando e Confortando, da Universidade Federal de Santa Catarina. Florianópolis - SC. Brasil. Email: telmacarraro@nfr.ufsc.br
} 


\section{INTRODUÇÃO}

A promoção da saúde é uma das estratégias para buscar a melhoria da qualidade de vida da população. Seu objetivo é desenvolver a gestão compartilhada entre usuários, movimentos sociais, trabalhadores do setor sanitário e de outros setores, produzindo autonomia e corresponsabilidade. ${ }^{1} 0$ ideal de promoção da saúde abarca o fortalecimento da capacidade individual e coletiva em saber conviver com as multiplicidades dos condicionantes da saúde. Este fortalecimento da saúde dá-se por meio da capacidade de escolha, da utilização do conhecimento com o discernimento de perceber as diferenças e semelhanças dos acontecimentos geradores de saúde ou de doença. ${ }^{2}$

Dentro dessa perspectiva, "as instituições de saúde são corresponsáveis pela promoção da saúde, não apenas de seus usuários, mas também de sua equipe multiprofissional, devendo fomentar práticas de cuidar de si" 3:108 Logo, a premissa básica para cuidar do outro está na necessidade de Cuidar de si, sendo imprescindível que isso faça parte do cotidiano do profissional, bem como do futuro profissional, constituindo-se como um estilo de vida. ${ }^{4}$ A compreensão de que o equilíbrio entre o cuidar de si e o cuidar do outro deve estar presente nas ações dos profissionais da saúde implica necessariamente cuidar de si. Mesmo esta prática necessita ser instigada constantemente no meio acadêmico, contribuindo para a formação dos futuros profissionais de saúde, especialmente dos enfermeiros.

Até porque é no momento de formação que o aluno amplia a consciência de si e é capaz de reconhecer a singularidade do outro, e desta forma está se tornando apto a compreender a dimensão humana do cuidado de Enfermagem, a configurá-lo como um momento especial. ${ }^{5}$

A convivência acadêmica é muito importante na formação do futuro profissional, e um dos principais desafios, dos adolescentes e dos jovens adultos acadêmicos, é a sua preparação para o mercado de trabalho. 0 fato é que esta etapa demanda diversas modificações em suas estruturas sociais, familiares, cognitivas e pessoais. Tais modificações abrangem também a aquisição de novos hábitos cotidianos, que se alteram no decorrer das mudanças de rotina de vida. Além de assumir as atividades acadêmicas passam a viver afastados de suas famílias, muitas vezes assumindo novas responsabilidades nessa etapa vivencial. Assim, alguns costumes e hábitos são preservados, outros são adquiridos, ou repadronizados, e alguns são abandonados, podendo transformar estruturas corporais e comportamentais dos acadêmicos.

Os hábitos são formados pela repetição de certos comportamentos (ações do cotidiano). Desse modo, depois que se aprende algo e se repete tal ação diversas vezes, o subconsciente passa a ser responsável por essa ação, não sendo mais necessário "pensar" para realizá-la, tornando-se, então, um hábito. ${ }^{6}$ Suas alterações são características das demandas da vida moderna, determinando novas adaptações, seja elas de ordem alimentar, de atividades físicas, de lazer e de cuidado, ou de des-cuidado, com a própria saúde.

Essas mudanças representam momentos de crise, os quais significam 0 amadurecimento e o fortalecimento, ou podem desencadear um desajuste e até mesmo o surgimento de doenças ${ }^{7}$, entre elas ressaltam-se o sobrepeso e a obesidade. 0 ajustamento sadio pode ser determinado pelo sucesso da pessoa na utilização de recursos para o atendimento de suas necessidades e pela sua capacidade para lidar com os sentimentos decorrentes do estresse.

As mudanças nos hábitos alimentares mobilizam as pessoas para uma dieta ocidentalizada, associada à diminuição progressiva da atividade física, apresentando como resultado o aumento de peso. ${ }^{8}$ Esta característica é observada não só na população em geral, mas também em adolescentes e adultos jovens, e principalmente em jovens em formação profissional, causada muitas vezes pelo excesso de atividades acadêmicas, sem conseguir estabelecer prioridades nessa fase da vida.

Assim, entende-se que a importância de cuidar de si perpassa, entre outras questões, pela atenção especial aos hábitos saudáveis, como a alimentação adequada e os exercícios físicos regulares, os quais contribuem para evitar o desenvolvimento do sobrepeso e da obesidade.

As consequências do desenvolvimento do sobrepeso e da obesidade podem ser condutores/precursores de doenças associadas, como hipertensão, diabetes, doenças cardíacas, entre outras, as quais comprometem a qualidade de vida do cuidador e do futuro cuidador profissional de Enfermagem. Vale ressaltar que o estresse, a ansiedade e a depressão são grandes causadores dessa disfunção, pois apresentam influência direta no comportamento alimentar. ${ }^{9} \mathrm{~A}$ obesidade é considerada uma epidemia mundial que atinge todas as idades e classes sociais sem discriminação. 0 conceito mais abrangente de obesidade é de uma síndrome multifatorial na qual a genética, o metabolismo e o ambiente interagem assumindo diferentes quadros clínicos nas diversas realidades socioeconômicas. ${ }^{10}$

0 desenvolvimento do sobrepeso e da obesidade configura-se em um processo de aumento de peso lento e gradativo, passando por diversos estágios de acúmulo de gordura no organismo, que pode ser localizada em determinadas partes ou no corpo inteiro. Esses estágios são classificados em sobrepeso e obesidade segundo o Índice de Massa Corporal (IMC), apresentado pela Organização Mundial da Saúde. ${ }^{11}$

Nesse contexto, conhecer os hábitos de vida dos acadêmicos, as suas percepções acerca da importância do cuidar de si, os fatores predisponentes ao sobrepeso e à obesidade presentes nos seus costumes de vidas e as relações desses elementos com o cuidado do outro é importante não só do ponto de vista da promoção da saúde, mas também para construção de vidas mais saudáveis e, neste sentido, tem chamado a atenção em nosso cotidiano. Afinal, viver bem e com 
saúde é um desejo de todos, mesmo que a concepção de bem viver e ter saúde não seja exatamente a mesma para todas as pessoas.

Diante deste contexto este artigo tem como objetivo identificar as percepções dos acadêmicos de enfermagem sobre o Cuidar de si, para a promoção da saúde e a evitabilidade do sobrepeso.

\section{MÉTODO}

Trata-se de uma pesquisa de abordagem qualitativa Convergente Assistencial (PCA) a qual requer que seja desenvolvida no espaço onde ocorrem as relações sociais. É compreendida e realizada em articulação com as ações que envolvem pesquisadores e demais pessoas representativas da situação a ser pesquisada em uma relação de cooperação mútua. ${ }^{12}$

Portanto, o lugar utilizado para o desenvolvimento desta pesquisa foi o Departamento de Enfermagem de uma Universidade Pública do Sul do Brasil, em uma sala de aula.

Participaram da pesquisa 42 acadêmicos, ou seja, $70 \%$ do total de alunos matriculados na $2^{\mathrm{a}}$ e $3^{\mathrm{a}}$ fases do curso. 0 critério para inclusão da participação foi que os acadêmicos estivessem devidamente matriculados nas fases referidas. Assim solicitaram-se aos acadêmicos interessados em participar da pesquisa sugestões de datas e os horários mais pertinentes para a realização dos encontros. Desta maneira iniciou-se o processo de aproximação com os acadêmicos, o qual foi importante e imprescindível para que eles pudessem conhecer a proposta da pesquisa. Após sugestões dos participantes em relação à data e horários, organizou-se um cronograma para os encontros. Nesse momento foi também esclarecido que seriam encontros realizados de forma descontraída, sendo que os acadêmicos poderiam vir com roupas confortáveis e não havia necessidade de material didático.

A coleta dos dados deu-se durante quatro dias não consecutivos, intitulados "Encontros de sensibilização e cuidado". Para a coleta, foram utilizadas dinâmicas de grupo composto por quatro a cinco alunos, confecções de cartazes, atividades lúdicas e de reflexão e utilização de um instrumento com perguntas abertas e fechadas, o qual continha dados de identificação pessoal/acadêmica, peso/altura e hábitos cotidianos. As atividades foram conduzidas pela própria mestranda, que tinha experiência neste tipo de atividade.

As falas foram gravadas em aparelho digital mp4 e posteriormente transcritas em diário de campo; vale ressaltar que cada fala gravada foi individualizada, para que pudesse apresentar clareza na gravação. 0 diário de campo foi estruturado por meio de colunas, as quais continham notas de observação, transcrição das falas, notas metodológicas e notas de cuidado realizado durante os encontros.

Em cada encontro foram abordados temas específicos: a importância do cuidar de si; o elo entre o cuidar de si, a promoção da saúde, a prevenção de doenças e evitabilidade do sobrepeso; hábitos saudáveis em seu cotidiano; e validação dos dados coletados. Nestes encontros formaram-se grupos diferentes com escolhas aleatórias para sua composição.

A análise dos dados seguiu os quatro processos genéricos da Pesquisa Convergente Assistencial: apreensão, síntese, teorização e transferência. Assim, foram lidos exaustivamente todos os dados contidos no diário de campo, e agruparam-se os dados por semelhança de significação, surgindo às seguintes categorias: Atividades físicas; Alimentação saudável; Ambiente familiar; e Autoestima e autoimagem.

Legalmente, foram cumpridas as determinações da Resolução n 196/96 do Conselho Nacional de Saúde. Assim, a inclusão dos acadêmicos de Enfermagem na pesquisa seguiu as determinações previstas nessa Resolução, que dispõe sobre as diretrizes e normas regulamentares da pesquisa envolvendo seres humanos, especialmente no que se refere ao Termo de Consentimento Livre e Esclarecido (TCLE). Foi garantida aos participantes, por meio do TCLE, a liberdade de participar ou não e de desistir a qualquer momento do estudo, como também foi assegurado o sigilo das informações e identidades, as quais foram apresentadas no estudo ora através das abreviações de seus nomes ora por denominações de grupos. 0 projeto foi avaliado pelo Comitê de Ética em Pesquisa da Universidade Federal de Santa Catarina, sendo aprovado sob o número 69/08.

\section{RESULTADOS E DISCUSSÃO}

As estratégias utilizadas nos encontros foram diversificadas para que pudesse ser focalizado o objetivo da pesquisa. Nesta perspectiva, os acadêmicos poderiam ficar a vontade para refletir e contextualizar os temas abordados, e desta forma emergiram as categorias que serão discutidas a seguir.

\section{Percepções quanto à Atividade física}

A partir dos dados analisados constatamos que os participantes fazem relações entre o cuidar de si e a saúde física, mental, como formas de estar e viver bem. Percebem, ainda, que as ações voltadas a cada área fazem parte de um conjunto de atividades para promoção da saúde e a evitabilidade do sobrepeso. Entretanto, demonstram suas dificuldades em relação ao desempenho das atividades físicas, como se apresenta nas falas abaixo:

(...) Faço academia, mas não aguento mais que dois meses, e, em relação à alimentação, só cuido quando estou me sentindo pesada. (A.C.O.B.)

(...) Fazia exercícios, mas há um ano parei, corria uma hora por dia. (S.M.G.) 


\section{(...) faço às vezes atividade física como caminhadas, e yoga parei há pouco tempo, mas pretendo retornar)... (E.C.Z.)}

Observa-se que os hábitos saudáveis relacionados ao exercício físico são reconhecidos por todos os acadêmicos, mas há evidências de que existe uma grande dificuldade em manter e persistir nesses hábitos em seu cotidiano, levando ao sedentarismo, ao aparecimento de doenças e ao possível aumento de peso.

Assim, é primordial que esses acadêmicos possam exercitar a reflexão como forma de apoio no que diz respeito à organização de seus hábitos cotidianos, para que estes sejam incorporados em suas vidas, e que o cuidar de si seja uma temática valorizada para o desenvolvimento profissional.

Sabe-se que os benefícios sociais, psicológicos e fisiológicos decorrentes da prática regular de exercícios são conhecidos, mas ainda muitas pessoas dizem não ter condições de se exercitar. As causas mais frequentes referidas para a não prática de exercícios físicos são a falta de tempo, poucas informações sobre os benefícios e sobre como se exercitar, e a carência de instalações adequadas e convenientes e fadiga geral associada a longas jornadas de trabalho. ${ }^{6}$

A atividade física é qualquer movimento corporal com gasto energético acima dos níveis de repouso. ${ }^{6} \mathrm{~A}$ prática desta atividade configura-se cada vez mais como uma importante medida eficaz e de baixo custo para proporcionar melhores condições de vida às pessoas. É reconhecida cientificamente como uma das possibilidades de ajuda terapêutica a diversas morbidades. ${ }^{13}$

Entendemos que a prática dos exercícios físicos contribui diretamente e indiretamente para a busca ativa da saúde e desta forma fortalece o poder vital, denotando-se como parte integrante do cuidar de si e da promoção da saúde. A prática de exercícios físicos constitui uma forma de relaxamento, de reposição das energias e da realização de atividade, que se faz com gosto para se obter prazer e descontração. ${ }^{3}$

\section{Percepção quanto à Alimentação}

Os participantes relatam que a a limentação é importante para viver saudável e é parte do cuidado de si, porém chamam atenção às dificuldades relacionadas à adoção de hábitos para tal. Podemos observar, pelas suas falas, as relações entre a alimentação e o cuidar de si

(...) cuidar da alimentação...tentamos comer bem às vezes [...] mas quando dá "manero" bastante nas gorduras, mas não deixo de comer. (2o grupo)

\section{(...) tento cuidar da alimentação, comendo frutas e verduras, e quantidades pequenas. (S.M.G.)}

\section{(...) tento manter uma boa alimentação (E.C.Z.)}

A tomada de consciência da importância da alimentação saudável revela que existe o empenho de manter os hábitos alimentares, porém há momentos em que não conseguem manter. Hábitos saudáveis de alimentação são diferentes em estruturas culturais, econômicas e políticas; assim se torna necessária maior atenção na promoção da saúde voltada, principalmente, para as políticas dirigidas aos determinantes desses hábitos, incluindo ações que subsidiem a produção de alimentos saudáveis, controle sobre propagandas que incentivem o consumo de alimentos densamente energéticos e ações concretas que incentivem a prática de

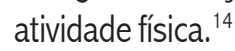

Neste sentido, observa-se que a alimentação não é um dos aspectos mais importantes para o cuidado de si, visto ser este hábito deixado de lado em decorrência do cotidiano atribulado. Alimentar-se bem e com qualidade envolve uma decisão interior; ou seja, cada pessoa é responsável por aquilo que decide comer em suas refeições, configurando-se este ato como importante para o cuidar de si e a promoção de sua própria saúde. $^{3}$

\section{Percepção no ambiente familiar}

Um ambiente participativo e a influência dos pais no estilo de vida dos filhos, incluindo a escolha dos alimentos, indicam o importante papel da família em relação ao ganho de peso infantil. 0 consumo muitas vezes é influenciado pelo nível socioeconômico familiar. ${ }^{15}$

Chamou a atenção quando um dos participantes revelou que "...só como salada porque a minha mãe me obriga... Mas eu como(A.P.S )". Parece que a relação da alimentação saudável pode estar vinculada a questões culturais em que a mãe, neste caso, tenta influenciar o hábito alimentar utilizando para isso o papel de autoridade na família.

A família vem constantemente passando por reformulações de conceitos e comportamentos e, atualmente, encontra-se em fase de restabelecimento. Contudo, seja pela forma como se apresente, a estrutura familiar pode desempenhar um papel fundamental na formação dos indivíduos. No que se refere ao comportamento, tende a reproduzir práticas com as quais a sociedade contemporânea se apresenta, ou seja, dentro das contradições e ambiguidade dos desejos. ${ }^{16}$

A respeito da promoção da saúde, alguns acadêmicos expressaram que as relações interpessoais vão além das afinidades familiares, demonstrando que outros apoios sociais são relevantes:

(...) Manter um bom relacionamento com a familia... Aprender a se conhecer, saber suas necessidades controlar as emoções, ter bons relacionamentos com os outros, ter humanidade ou qualquer outro tipo de relacionamento, cultivar a familia, fazer o que gosta ( $3^{\circ}$ grupo) 
(...) conversar com os amigos estar com a família, esporte, ter bons relacionamentos, meditar; a gente cuida da família, do marido, dos pacientes, mas primeiro a gente tem que cuidar da gente também (4ำ grupo)

É sugerido nestes depoimentos que o ambiente universitário propicia relações interpessoais, as quais auxiliam na formação não só acadêmica, mas também do caráter dos indivíduos em que as amizades, os relacionamentos amorosos são consolidados. Estas alianças são pautadas nas crenças e valores trazidos de suas famílias e na formação da personalidade.

Neste sentido, entende-se que cada família é única e singular e tem características e peculiaridades que acabam por definir o seu modo de ser e de viver em concordância com as possibilidades e limitações de cada um, compondo, assim, as características do indivíduo. ${ }^{17}$

As relações interpessoais ou familiares podem ter influência direta no cuidar de si, podendo ser um estímulo positivo na busca da promoção da saúde, principalmente por se tratar de jovens adultos em formação não só profissional, mas também pessoal. Como estratégia para cuidar de si, na medida do possível, devem conviver com pessoas que façam bem, no âmbito familiar ou social. ${ }^{3}$

\section{Percepção quanto à autoimagem e autoestima}

Há também que se registrar as relações intrínsecas entre o cuidar de si, alimentação saudável, prática de exercício físico e a autoimagem e a autoestima. Assim, foi manifestado em alguns depoimentos que a autoimagem está relacionada com o sobrepeso:

(...) dormir pouco, mal, engorda... Por isso que a gente está deste tamanho... (P.B.)

(...) 0 que mais gosto de mim é a saboneteiraa ${ }^{a}$ que é a parte mais magra do meu corpo (A.P.).

(...) Cuidar do corpo, se exercitar, se alimentar... (B.S.)

E em outros dados surge a autoestima:

(...) 0 que mais me apavora é a solidão... Não gosto de ficar sozinha $(A$.

\section{(...) Dou minha total atenção quando o momento é importante para mim, pois gosto de perfeição quando me refiro a tudo que faço (G.)}

Estas falas apresentam a associação com o peso, autoimagem e autoestima, pois a aparência corporal determina em grande parte os relacionamentos, as oportunidades sociais e profissionais, bem como as atitudes e reações diante dos desafios impostos pela vida. Além da imagem que temos perante as pessoas existe também a concepção que nós temos sobre nossa imagem, assim a concepção interna e subjetiva do próprio corpo é denominada imagem corporal. ${ }^{18}$

Quando as acadêmicas expressam que "Os outros dizem que eu não sou gorda, mas eu sou sim (G.)" ou que "Diante de um espelho eu me vejo enorme... (P.), eles deixam transparecer que a forma do corpo e o próprio peso são importantes referências de imagem corporal que fazem de si mesmas e esta referência a si própria busca a inclusão no grupo e na própria sociedade.

A dimensão pessoal dos mecanismos de autorregulação exerce um papel fundamental nos mecanismos de autoavaliação como: autoestima e autoconceito, os quais se referem à avaliação que o indivíduo faz de si, em diferentes áreas de atuação, e a apreciação em relação a si mesmo. ${ }^{18}$

Assim, surge 0 autoconceito das acadêmicas quando fazem as colocações acima referidas. Mas na mesma linha de entendimento, autoestima e autoconceito estão embutidos na questão da imagem corporal relacionada com o peso.

Ao olharmos os dados coletados nesta pesquisa, podemos perceber que as falas dos participantes apresentam contrapontos, pois por vezes colocam a importância de uma vida saudável, enfatizando os hábitos de alimentação saudável e exercícios físicos, porém não o fazem com regularidade, e ao mesmo tempo percebem seu corpo com peso acima do desejado. Entretanto, analisando as idades dos participantes e a formação ética, cognitiva, social e os hábitos, observa-se que em sua maioria ainda estão adolescentes e se encaminhando para uma vida adulta.

Os fatores psicológicos, como o controle, a percepção de si, a ansiedade e o desenvolvimento emocional de adolescentes podem contribuir para o aumento do sobrepeso e da obesidade e vice-versa. ${ }^{18}$

A reflexão e sensibilização sobre o cuidar de si e a promoção da saúde no cotidiano acadêmico pode não ser de grande importância. No entanto, esta discussão neste momento de vida dos acadêmicos aponta para uma possibilidade de transformação de seus hábitos na busca de um viver saudável. É evidente que os acadêmicos participantes desta pesquisa estão ainda revendo e elaborando seus próprios estilos de vida, fazendo suas escolhas e condutas diante das suas concepções, porém não deixando de expressar a influência que suas famílias exercem.

\section{CONSIDERAÇÕES FINAIS}

Os achados encontrados neste estudo apontam para o reconhecimento dos acadêmicos sobre a importância de cuidar de si; apresentam conhecimento sobre os hábitos saudáveis, porém há evidências de que existem grandes dificuldades em manter e persistir nesses hábitos, propiciando o sedentarismo, 
0 aparecimento de doenças e 0 aumento de peso; relacionam as ações de promoção da saúde com os hábitos saudáveis, mas demonstram que essas ações estão distantes de seus cotidianos; percebem que o cuidar de si é uma forma de manter o peso e/ ou buscar o peso adequado.

Também ficou claro que pensar sobre a realidade, de forma crítica e criativa, é uma forma de buscar a reflexão e a transformação de alguns conceitos do dia-a-dia, em relação à promoção da saúde e ao cuidar de si. Por outro lado, sensibilizar para a importância do cuidar de si na construção de uma vida saudável implica buscar a reflexão sobre seus próprios hábitos e o enfrentamento das situações adversas para mudá-las, se necessário. Isso se dá por meio do estabelecimento de relações afetivas e solidárias, adotando a postura de "ser e estar no mundo" com o objetivo de viver bem. É agir em favor de uma saúde que se faz e se melhora a cada dia. É promover a saúde, cuidando de si para cuidar do outro.

Consideramos que uma das grandes contribuições desta pesquisa para os profissionais da saúde, em especial para os enfermeiros e futuros enfermeiros, está na apresentação de subsídios para novas reflexões sobre a realidade dos acadêmicos de Enfermagem, na relação aos seus hábitos cotidianos, na busca de um estilo de vida mais saudável, caracterizando-os, dessa forma, como profissionais que sabem cuidar de si para cuidar do outro.

\section{REFERÊNCIAS}

1. Ministério da Saúde(BR). Portaria nº687, de 30 de março de 2006. Aprova a Política Nacional de Promoção da Saúde. Disponível em: < http:// 64.233.169.132/search?q=cache:fasy23iN4EQJ:dtr2004.saude.gov.br/ dab/docs/legislacao/portaria_687_30_03_06.pdf+Portaria+687,\&hl=pt-

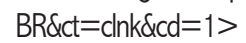

2. Czeresnia D, Freitas CM, organizadoras. Promoção da saúde: conceitos, reflexões e tendências. Rio de Janeiro: Fiocruz; 2003.

3. Radünz V. Uma filosofia para enfermeiros: o Cuidar de si, a convivência com a finitude e a evitabilidade do burnout. Florianópolis: Programa de Pós-graduação em Enfermagem, Universidade Federal de Santa Catarina; 2001.

4. Kroeger MIB. Fatores que influem no trabalho do terapeuta natural durante 0 atendimento de trabalhadores com depressão [dissertação]. Florianópolis : Universidade Federal de Santa Catarina; 2003.

5. Kestenberg CCF, Reis MAS, Motta WC. et al. Cuidando do estudante e ensinando relações de cuidado de Enfermagem. Texto\&Contexto Enferm. 2006; 15 (n esp):193-200.

6. Nahas MV. Atividade física, saúde e qualidade de vida: conceitos e sugestões para um estilo de vida ativo. Londrina: Miograf; 2006.
7. Jorge MSB, Rodrigues ARF. Serviços de apoio ao estudante oferecidos pelas escolas de Enfermagem no Brasil. Rev Latino-am Enfermagem. 1995; 3(2): 59-68.

8. Sebold LF, Radünz V, Rocha PK. et al. Obesidade e cuidado. In: Martini JG, Kalinowski CE. Programa de Atualização em Enfermagem-PROENF: saúde do adulto. Porto Alegre: Artmed/Panamericana; 2007.

9. Vasques F, Martins FC, Azevedo AP. Aspectos psiquiátricos do tratamento da obesidade. Rev Psiq Clin. 2004; 31(4): 195-98.

10. Murate EH. Atenção dietética e atividade física no tratamento de pessoas obesas e/ou sobrepeso. [citado 2007 jan 23]. Disponível em: <http://www.proec.ufpr.br/enec2005/ download/pdf/SA\%DADE/pdf\%20 saude/4 $7 \% 20$ -

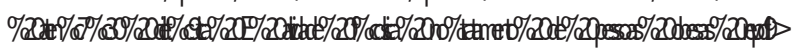

11. Organização Mundial da Saúde-OMS. Obesity and overweight. [citado 2006 out 5]. Disponivel em: < http://www.who.int/dietphysicalactivity/ publications/fact/obesity/em 2002>

12. Trentini M, Paim L. Pesquisa em enfermagem: uma modalidade convergente-assistencial. $2^{\mathrm{a}}$ ed. Florianópolis: UFSC; 2004.

13. Siqueira FCV, Nahas MV, Facchini LA. et al. Atividade física em profissionais de saúde do sul e nordeste do Brasil. Cad Saude Publica. 2009 set; 25(9):1917- 928.

14. Vinholes DB, Assunção MCF, Neutzling MB. Freqüência de hábitos saudáveis de alimentação medidos a partir dos Dez passos da alimentação saudável do Ministério da Saúde. Pelotas, Rio Grande do Sul, Brasil. Cad Saude Publica. 2009 abr; 25(4): 791-99.

15.Sichieri R, Souza RA. Estratégias para prevenção da obesidade em crianças e adolescentes. Cad Saude Publica. 2008; 24(supl 2): 209-34.

16. Stenzel LM. A influência da imagem corporal no desenvolvimento e na manutenção dos transtornos alimentares. In: Nunes MA, Apolinário JC, Galvão AL, Coutinho W. Transtornos alimentares e obesidade. $2^{\mathrm{a}}$ ed. Porto Alegre: Artmed; 2006.

17. Schaurich D. Compreensões de acadêmicos de enfermagem sobre famílias: algumas reflexões. Esc Anna Nery. 2009 abr/jun; 13 (2): 415-20.

18. Carvalho AMP, Cataneo C, Galindo EMC, Malfará CT. Auto conceito e imagem corporal em crianças obesas. Paidéia: cadernos de psicologia e educação. 2005 jan/abr; 15(30).

\section{NOTA}

aParte do osso da clavícula que fica evidenciado quando a massa corporal é diminuta. 